\title{
EXAME CITOPATOLÓGICO DO COLO DO ÚTERO: INVESTIGAÇÃO SOBRE O CONHECIMENTO, ATITUDE E PRÁTICA DE GESTANTES
}

\author{
Ana Raquel Rodrigues Rosa1, Tayane Siqueira de Lima e Silva², Izabel Cristina da Silva Carvalho², \\ Antônia Sylca de Jesus Sousa ${ }^{3}$, Ângelo Brito Rodrigues ${ }^{4}$, Jardeliny Corrêa da Penha ${ }^{5}$
}

\begin{abstract}
RESUMO: Objetivo: verificar o conhecimento, a atitude e a prática das gestantes atendidas na Estratégia Saúde da Família sobre o exame citopatológico do colo do útero. Método: inquérito Conhecimento, Atitude e Prática desenvolvido com 46 gestantes atendidas em unidades básicas de saúde de Floriano, estado do Piauí. A coleta de dados foi realizada de maio a julho de 2016, com aplicação de um instrumento previamente elaborado. Realizaram-se análise descritiva, e, para associação das variáveis nominais, o Teste de Qui-quadrado. Resultados: as gestantes apresentaram percentual de conhecimento, atitudes e práticas inadequado sobre o exame citopatológico. Houve associação significante de algumas variáveis com a prática das gestantes em relação ao citopatológico do colo uterino (exame pode ser realizado durante a gestação, $p=0,030$, motivo pelo qual faria o exame estando grávida, $p=0,043)$. Conclusão: as gestantes precisam conhecer o exame citopatológico do colo do útero e a importância da sua realização durante a gestação, para conseguirem aderir à prática do exame.
\end{abstract}

DESCRITORES: Teste de papanicolaou; Conhecimentos, atitudes e prática em saúde; Gestantes; Neoplasias do colo do útero; Saúde da mulher; Atenção primária à saúde.

\section{CERVICAL CYTOLOGY EXAMINATION: INQUIRY INTO THE KNOWLEDGE, ATTITUDE AND PRACTICE OF PREGNANT WOMEN}

\begin{abstract}
Objective: to verify the knowledge, attitude and practice regarding the cervical cytology examination of pregnant women assisted in the Family Health Strategy. Method: Knowledge, Attitude and Practice inquiry developed with 46 pregnant women attended in primary health units of Floriano, Piauí state. Data collection was carried out from May to July 2016, with the application of a previously elaborated instrument. Descriptive analysis was performed, with the Chi-square test for the association of the nominal variables. Results: the pregnant women presented inadequate knowledge, attitudes and practices regarding the cytology examination. There was a significant association of some variables with the practice of pregnant women in relation to cervical cytology (examination can be performed during pregnancy, $p=0.030$, reason why the test would be performed during pregnancy, $p=0.043$ ). Conclusion: pregnant women need to understand the cervical cytology examination and the importance of its performance during pregnancy, in order to be able to adhere to the practice of performing the examination.
\end{abstract}

KEYWORDS: Papanicolaou test; Knowledge, Health attitudes and practice; Pregnant women; Neoplasms of the cervix; Women's health; Primary healthcare.

\section{EXAMEN CITOPATOLÓGICO DEL CUELLO DEL ÚTERO: INVESTIGACIÓN SOBRE EL CONOCIMIENTO, ACTITUD Y PRÁCTICA DE MUJERES EMBARAZADAS}

RESUMEN: Objetivo: verificar el conocimiento, la actitud y la práctica de las embarazadas atendidas en la Estrategia Salud de la Familia sobre el examen citopatológico del cuello del útero. Método: encuesta "Conocimiento, Actitud y Práctica" desarrollada con 46 embarazadas atendidas en unidades básicas de salud en el municipio de Floriano, estado de Piauí. El recogimiento de datos fue realizado de mayo a julio de 2016, con aplicación de un instrumento previamente elaborado. Se realizaron análisis descriptivos; y, para la asociación de las variables nominales se utilizó el test de Chi-cuadrado. Resultados: las embarazadas presentaron un porcentaje de conocimiento, actitudes y prácticas inadecuado sobre el examen citopatológico. Hubo asociación significativa de algunas variables con la práctica de las embarazadas, en relación al citopatológico del cuello uterino (el examen puede ser realizado durante la gestación, p=0,030; motivo por el cual harían el examen estando embarazadas, $p=0,043)$. Conclusión: las embarazadas precisan conocer el examen citopatológico del cuello del útero y la importancia de su realización durante la gestación, para conseguir adherir a la práctica del examen.

DESCRIPTORES: Test de Papanicolaou; Conocimientos, actitudes y práctica en salud; Gestantes; Neoplasias do cuello del útero; Salud de la mujer; Atención primaria a la salud.

'Enfermeira. Graduada em Enfermagem pela Universidade Federal do Piauí (UFPI). Floriano-PI-Brasil.

Enfermeira. Graduada em Enfermagem pela Universidade Federal do Piauí (UFPI). Teresina-PI-Brasil.

${ }^{3}$ Enfermeira. Mestre em Cuidados Clínicos em Enfermagem e Saúde. Professora Temporária do Departamento de Enfermagem da Universidade Estadual do Piauí. Picos-PI-Brasil.

${ }^{4}$ Enfermeiro. Mestre em Saúde Pública. Docente do Departamento de Enfermagem da Universidade Federal do Piauí. Floriano-PI-Brasil. ${ }^{5}$ Enfermeira. Doutora em Enfermagem pela Universidade Federal do Ceará. Docente do Departamento de Enfermagem da Universidade Federal do Piauí. Foriano-PI-Brasil. 


\section{INTRODUÇÃO}

O câncer do colo do útero (CCU) é considerado um importante problema de saúde pública, em decorrência da elevada morbimortalidade. Embora ao longo das últimas três décadas tenha se reduzido a taxa de incidência desse agravo, em dados mundiais de 2012 foi estimada a ocorrência de 527 mil casos novos em mulheres, sendo este o quarto tipo de câncer mais comum entre elas ${ }^{(1)}$.

No Brasil, no ano de 2016, foram esperados 16.340 casos novos de CCU, com risco estimado de 15,85 casos a cada 100 mil mulheres. Na Região Nordeste, esse câncer ocupou a segunda posição, com um risco de 19,49 para cada 100 mil mulheres. No Piauí, a estimativa de casos novos foi de 24,51, e, especificamente em Teresina, de 30,32, a cada 100 mil mulheres, estando acima da média nacional(1).

Quanto aos métodos de prevenção primária ao CCU, tem-se o uso de preservativos, que protege parcialmente do contágio pelo Papiloma vírus humano (HPV), além das vacinas contra o HPV: a bivalente, que protege contra os tipos oncogênicos 16 e 18, e a quadrivalente, contra os tipos não oncogênicos 6 e 11 e oncogênicos 16 e 18. Ambas as vacinas são disponíveis, em território brasileiro, gratuitamente para indivíduos com idade entre 9 e 13 anos $^{(2)}$.

Sobre os métodos de rastreamento do CCU e das lesões intraepiteliais, cita-se o exame citopatológico do colo do útero (ou teste de Papanicolaou), estratégia de busca precoce, realizado na Estratégia Saúde da Família (ESF), pelo enfermeiro ou médico ${ }^{(2-3)}$. As diretrizes para o rastreamento do CCU recomendam a realização anual do exame em mulheres na faixa etária de 25 a 64 anos, e com intervalo de 3 anos após dois exames consecutivos anuais negativos ${ }^{(2,4)}$.

Nas gestantes, também deve ser realizado o exame citopatológico do colo do útero, o qual pode ser solicitado como um dos exames complementares, preferencialmente até o sétimo mês de gestação ${ }^{(2)}$. A presença espontânea da mulher gestante na ESF, período em que vai com mais frequência aos serviços de saúde, pode ser aproveitada com a finalidade de detectar da patologia(5). É válido ressaltar que não há necessidade de ser realizada a coleta da endocérvice nas gestantes, visto que a junção escamocolunar está exteriorizada ${ }^{(2)}$.

Destarte, obedecendo às recomendações do Ministério da Saúde, observa-se quão importante é que todas as mulheres, especialmente as grávidas, sejam submetidas ao exame citopatológico. É primordial que elas detenham conhecimento sobre o exame, bem como tenham atitude e prática necessárias para realizá-lo, a fim de que haja rastreamento e controle efetivos do CCU no Brasil.

A avaliação do conhecimento, da atitude e da prática da população feminina, especialmente das grávidas, sobre o exame citopatológico do colo do útero tem como finalidade observar a percepção que as mulheres possuem sobre o CCU e sobre o exame que o detecta, o que pode ter grande influência na sua realização e na frequência com que é realizado. Essas informações podem servir de embasamento para os profissionais e os serviços de saúde, no que diz respeito à adoção de medidas que possam garantir informações e saúde àquela população.

Em face do exposto, o presente estudo teve como objetivo verificar o conhecimento, a atitude e a prática das gestantes atendidas na Estratégia Saúde da Família de Floriano, estado do Piauí, sobre o exame citopatológico do colo do útero. A importância da pesquisa consiste no fortalecimento de ações de promoção da saúde e de prevenção do CCU voltadas às gestantes. Proporcionar sensibilização pode romper mitos que impedem a realização do exame durante o período gestacional e, assim, promover maior aceitação para realizá-lo, melhorando o controle e o rastreamento dessa patologia.

\section{METODOLOGIA}

Trata-se de um estudo quantitativo, do tipo inquérito Conhecimento, Atitude e Prática (CAP), o qual consiste em uma avaliação formativa, com um conjunto de questionamentos que visam avaliar o que as pessoas sabem, pensam e como atuam diante de um determinado problema ${ }^{(6)}$. 
A pesquisa foi realizada em seis Unidades Básicas de Saúde (UBS) do município de Floriano, estado do Piauí. Essas UBS foram selecionadas porque possuíam maior quantitativo de gestantes cadastradas, somadas totalizavam 222 mulheres grávidas, assim, a amostragem se deu por conveniência. A população foi constituída por gestantes cadastradas e atendidas nesse serviços, e o número de participantes foi definido por meio do cálculo amostral para população finita, o qual gerou um tamanho amostral de 198 gestantes.

As participantes obedeceram aos seguintes critérios de inclusão: mulheres com idade gestacional de até 28 semanas ( $7^{\circ}$ mês), contada a partir da data da última menstruação (DUM), e que tivessem realizado pelo menos uma consulta pré-natal. Os critérios de exclusão foram: mulheres com idade inferior a 18 anos e as que apresentassem dificuldade para entender os questionamentos. A partir da instituição desses critérios, a amostra final foi de 46 gestantes. Pôde-se perceber a ausência dessas mulheres nas UBS para o acompanhamento pré-natal.

A coleta de dados ocorreu no período de maio a julho de 2016. Inicialmente, as gestantes eram convidadas a participar do estudo enquanto aguardavam a realização de qualquer consulta ou atendimento nas UBS. Direcionadas a uma sala reservada para a entrevista estruturada e individual, as participantes, esclarecidas sobre os objetivos da pesquisa, assinaram em duas vias o Termo de Consentimento Livre e Esclarecido (TCLE).

Para nortear a coleta de dados, utilizou-se de um instrumento, que compreendia, na primeira parte, questões sobre as variáveis sociodemográficas (idade, estado civil, escolaridade e renda individual mensal) e gineco-obstétricas (início de atividade sexual, história prévia de doença/infecção sexualmente transmissível - DST/IST, idade gestacional e número de gestações); e, na segunda, o inquérito CAP, adaptado de três versões de inquéritos já aplicados em diferentes grupos femininos ${ }^{(7-9)}$.

O inquérito CAP consistiu em um formulário que continha questões objetivas sobre o conhecimento, a atitude e a prática das mulheres sobre o exame citopatológico do colo do útero. Neste estudo, consideraram-se como adequadas as seguintes especificações: conhecimento - a gestante ouviu falar sobre o exame e soube relatar que sua finalidade é detectar o CCU, afirmar que pode ser realizado durante a gestação e citar dois cuidados (recomendações prévias) que devem ser executados antes do procedimento; atitude - respondeu que realizaria o exame durante a gestação e relatou que o faria para detectar o CCU; e prática - a participante respondeu que realizou o exame durante a gestação atual ou anterior.

Os dados foram tabulados e analisados pelo programa estatístico Statistical Package for Social Sciences IBM (SPSS IBM), versão 20.0. Realizou-se análise descritiva (frequências absolutas e relativas), e para associação das variáveis nominais executou-se o Teste de Qui-quadrado, adotando-se um valor de p menor que 0,05 e intervalo de confiança de 95\%.

A pesquisa foi submetida e aprovada pelo Comitê de Ética em Pesquisa, com parecer n. 1.632.521, de modo a garantir os princípios expressos nas Resoluções n. 466/12 e 510/16 do Conselho Nacional de Saúde (CNS) ${ }^{(10-11)}$, que tratam das pesquisas com seres humanos. Isto garantiu o sigilo, o anonimato e o direito de as gestantes deixarem de participar da pesquisa a qualquer momento.

\section{RESUltADOS}

Participaram do presente estudo 46 gestantes, com idade média de 24,65 anos, escolaridade média de 13,07 anos de estudo, estado civil e média de $R \$ 308,53$ de renda individual mensal. Verificou-se que 23 (50\%) possuíam entre 21 e 30 anos de idade, 21 (45,7\%), entre 13 e 15 anos de estudo, e 21 $(45,7 \%)$ se encontravam em união estável. No concernente à renda individual mensal, 24 (52,1\%) não tinham rendimento e $10(21,7 \%)$ possuíam uma renda de meio a um salário mínimo.

Quanto às variáveis gineco-obstétricas, observou-se que $36(78,2 \%)$ gestantes haviam iniciado atividade sexual entre 15 e 19 anos de idade, 43 (93,5\%) não continham história prévia de DST/IST, 26 $(56,5 \%)$ apresentavam idade gestacional entre 14 e 26 semanas, e $24(52,1 \%)$ mencionaram duas a três gestações. 
De acordo com o Gráfico 1, as mulheres possuíam predominantemente conhecimento, atitude e prática inadequados, respectivamente, 44 (95,7\%), 45 (97,8\%) e $38(82,6 \%)$.

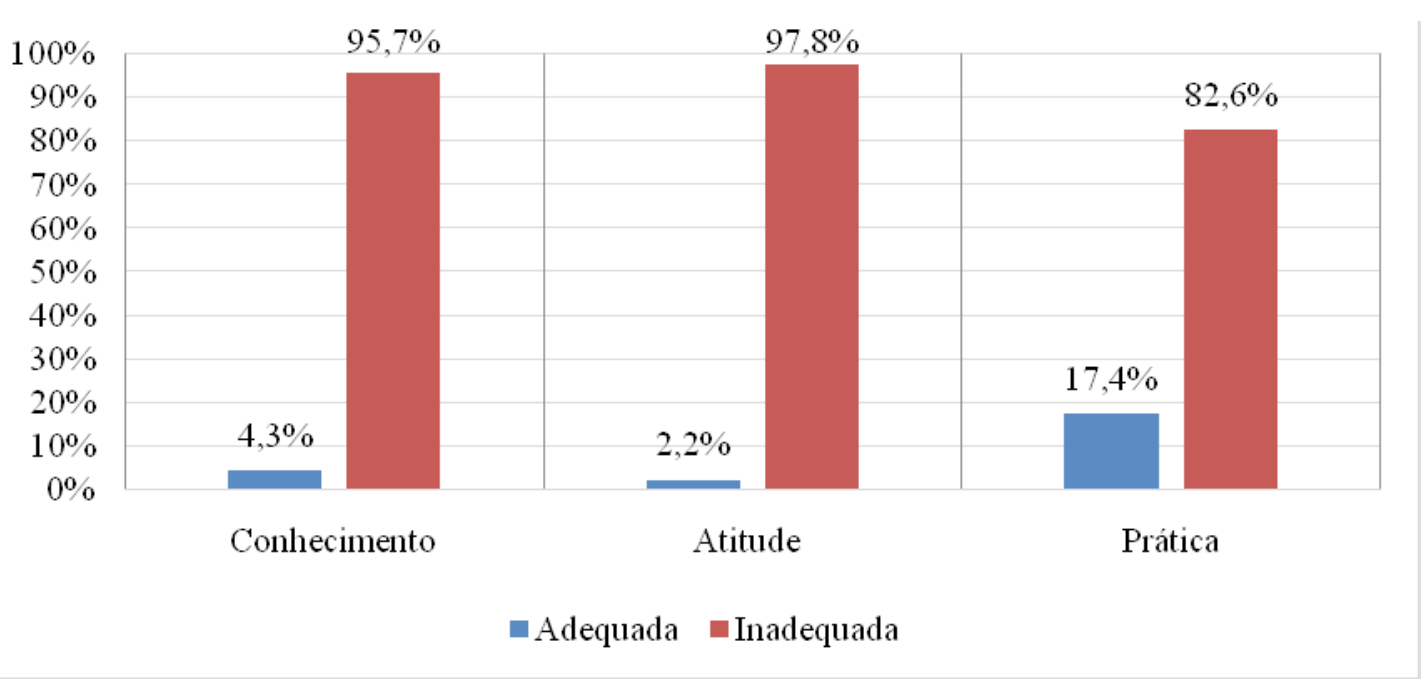

Gráfico 1 - Descrição do conhecimento, atitude e prática das gestantes sobre o exame citopatológico do colo do útero. Floriano, PI, Brasil, 2016

Associou-se o conhecimento e a atitude das gestantes à prática adequada ou inadequada em relação ao exame citopatológico do colo do útero. Como evidenciado, na Tabela 1, a variável conhecimento que as questionava se o exame poderia ser realizado durante a gestação $(p=0,030)$ e uma atitudinal (motivo pelo qual faria o exame estando grávida, $p=0,043$ ) tiveram associação estatisticamente significativa com a prática.

Tabela 1 - Associação da prática do exame citopatológico do colo do útero com o conhecimento e a atitude das gestantes sobre o exame. Floriano, PI, Brasil, 2016

\begin{tabular}{|c|c|c|c|c|c|}
\hline \multirow{3}{*}{$\begin{array}{c}\text { CONHECIMENTO E ATITUDE DAS GESTANTES } \\
\text { ACERCA DO EXAME }\end{array}$} & \multicolumn{4}{|c|}{ PRÁTICA DAS GESTANTES SOBRE O EXAME } & \multirow{3}{*}{ p-valor } \\
\hline & \multicolumn{2}{|c|}{ ADEQUADA } & \multicolumn{2}{|c|}{ INADEQUADA } & \\
\hline & $\mathbf{N}$ & $\%$ & $\mathbf{N}$ & $\%$ & \\
\hline \multicolumn{6}{|l|}{ Já ouvi falar do exame $(n=46)$} \\
\hline Sim & 8 & 17,4 & 35 & 76,1 & \multirow{2}{*}{0,411} \\
\hline Não & - & - & 3 & 6,5 & \\
\hline \multicolumn{6}{|l|}{ O exame pode ser realizado durante a gestação $(n=46)$} \\
\hline Sim & 8 & 17,4 & 23 & 50,0 & \multirow{2}{*}{0,030} \\
\hline Não & - & - & 15 & 32,6 & \\
\hline \multicolumn{6}{|l|}{ Faria o exame durante a gestação $(n=46)$} \\
\hline Sim & 8 & 17,4 & 32 & 69,6 & \multirow{2}{*}{0,228} \\
\hline Não & - & - & 6 & 13,0 & \\
\hline \multicolumn{6}{|l|}{ Por que faria o exame $(n=40)$} \\
\hline Outros motivos* & 7 & 17,5 & 32 & 80,0 & \multirow{2}{*}{0,043} \\
\hline Prevenir o CCU & 1 & 2,5 & - & - & \\
\hline
\end{tabular}

*Outros motivos: detectar inflamação; prevenir doenças em geral; evitar infecção no feto ou durante o parto; proteger o bebê; saber se tem alguma alteração; e porque é importante. 
Considerando que o conhecimento é uma variável fundamental para o desenvolvimento de uma prática adequada no cuidado à saúde, foi possível também relacionar, por análise bivariada simples, as variáveis sobre conhecimento com a prática adequada ou inadequada das gestantes quanto ao exame citopatológico do colo do útero. As informações são apresentadas na Tabela 2.

Tabela 2 - Distribuição da frequência da prática do exame citopatológico do colo do útero segundo as variáveis relacionadas ao conhecimento das gestantes sobre o exame. Floriano, PI, Brasil, 2016

\begin{tabular}{|c|c|c|c|c|}
\hline \multirow{3}{*}{ CONHECIMENTO DAS GESTANTES ACERCA DO EXAME } & \multicolumn{4}{|c|}{$\begin{array}{l}\text { PRÁTICA DAS GESTANTES SOBRE } \\
\text { O EXAME }\end{array}$} \\
\hline & \multicolumn{2}{|c|}{ ADEQUADA } & \multicolumn{2}{|c|}{ INADEQUADA } \\
\hline & $\mathbf{N}$ & $\%$ & $\mathbf{N}$ & $\%$ \\
\hline \multicolumn{5}{|l|}{ Para que serve o exame/Finalidade $(n=46)$} \\
\hline Prevenir o CCU & 2 & 25 & 4 & 10,5 \\
\hline Detectar o CCU & - & - & 6 & 15,8 \\
\hline Não sabe & 1 & 12,5 & 15 & 39,5 \\
\hline Outras finalidades & 5 & 62,5 & 13 & 34,2 \\
\hline \multicolumn{5}{|l|}{ Cuidados antes do exame $(n=46)$} \\
\hline Apenas não ter relação sexual 24h antes & 1 & 12,5 & 4 & 10,5 \\
\hline Apenas aparar os pelos pubianos & 1 & 12,5 & 7 & 18,4 \\
\hline Não ter relação sexual 24h antes e aparar os pelos pubianos & 1 & 12,5 & 2 & 5,3 \\
\hline $\begin{array}{l}\text { Não ter relação sexual } 24 \mathrm{~h} \text { antes e nem usar duchas e cremes vaginais } 48 \mathrm{~h} \\
\text { antes }\end{array}$ & 1 & 12,5 & 1 & 2,6 \\
\hline Não sabe/não lembra & 4 & 50 & 24 & 63,2 \\
\hline \multicolumn{5}{|l|}{ Fonte de conhecimento sobre o exame $(n=46)$} \\
\hline Familiares & 1 & 12,5 & 10 & 26,3 \\
\hline Enfermeiro & 3 & 37,5 & 5 & 13,2 \\
\hline Agente Comunitário de Saúde & 1 & 12,5 & 1 & 2,6 \\
\hline Familiares e Enfermeiro & - & - & 1 & 2,6 \\
\hline Vizinha/amiga & - & - & 5 & 13,2 \\
\hline Televisão & - & - & 4 & 10,5 \\
\hline Palestras/cartazes & 2 & 25 & 8 & 21,1 \\
\hline \multicolumn{5}{|l|}{ Por que o exame não pode ser realizado durante a gestação $(n=15)$} \\
\hline Não sabia responder & 1 & 25 & 2 & 18,2 \\
\hline Prejudica o bebê (feto) ou a gestação & - & - & 7 & 63,6 \\
\hline Pode causar alteração hormonal & 2 & 50 & 1 & 9,1 \\
\hline Pode ocasionar aborto & 1 & 25 & 1 & 9,1 \\
\hline
\end{tabular}

Embora oito das 46 gestantes indagadas sobre a finalidade do exame citopatológico do colo do útero possuíssem prática adequada, nenhuma relatou que era para detectar o CCU. Cinco (62,5\%) citaram outras finalidades, três $(16,66 \%)$ inflamação, duas $(11,11 \%)$ saber se tem nódulo ou cisto no útero, uma $(5,55 \%)$ infecção, cinco $(27,8 \%)$ detectar uma doença, seis $(33,33 \%)$ saber se tem doença no útero, uma (5,55\%) saber se tem câncer, seguidas de 16 (34,9\%), que não sabiam o motivo pelo qual o exame era realizado. Entre as 38 que tinham prática inadequada, 15 (39,5\%) delas não souberam informar a finalidade do exame citopatológico. 
Sobre os cuidados a serem tomados antes do exame, a maioria das gestantes que possuía prática adequada não sabia ou não se lembrava de recomendações prévias, e entre aquelas com prática inadequada, esta variável também foi predominante, com $24(63,2 \%)$ respostas.

No que tange à fonte de conhecimento sobre o citopatológico, entre as gestantes com prática adequada, as informações foram predominantemente recebidas de enfermeiros e cartazes/palestras. Entre as participantes com prática inadequada, grande parte $(10,6,3 \%)$ ouviu falar do exame pelos familiares, e oito $(21,1 \%)$ a partir de cartazes/palestras.

Das 46 gestantes, 15 afirmaram que o exame citopatológico do colo do útero não pode ser realizado durante a gestação. Destas, quatro possuíam prática adequada do exame, porém duas delas afirmaram erroneamente que este poderia causar alteração hormonal. Entre $\mathbf{1 1}$ gestantes que tinham prática inadequada, sete $(63,6 \%)$ afirmaram que o exame prejudicaria o bebê (feto) ou a gestação.

\section{DISCUSSÃO}

A faixa etária prevalente entre as gestantes entrevistadas corresponde à de mulheres com um perfil de adultas jovens. Pesquisa sobre a cobertura do exame citopatológico do colo do útero durante o prénatal, realizada com 445 puérperas em Rio Grande, estado do Rio Grande do Sul, revelou que 141 $(31,7 \%)$ tinham de 25 a 29 anos de idade; além disso, o grupo de puérperas com idade igual ou superior a 20 anos apresentou prevalência $(p=0,004)$ de realização do exame nos últimos 36 meses, quando comparadas às mulheres de menor idade ${ }^{(12)}$.

No que concerne ao estado civil, notou-se que a maior parte das participantes possuía união estável. Esse dado assemelha-se ao do estudo desenvolvido em São Gonçalo do Amarante, estado do Ceará, com 390 mulheres que aguardavam a realização do citopatológico, visto que 302 (77,4\%) delas estavam em união estável ${ }^{(13)}$.

Em ensaio comunitário, realizado com 60 mulheres cadastradas em uma equipe da ESF da cidade de Montes Claros, estado de Minas Gerais, que investigou a adesão destas ao exame citopatológico do colo do útero, foi observado que 38 (63,3\%) eram casadas, e essa variável mostrou associação estatisticamente significativa com a adesão ao exame $(p=0,014)^{(14)}$. Isso não corrobora o que foi encontrado na presente pesquisa, pois, embora a maioria estivesse em união estável, não houve prática adequada à realização do citopatológico durante a gestação, conforme ilustra o Gráfico 1.

Sobre a escolaridade, de modo predominante as participantes possuíam de 13 a 15 anos de estudo. Achado discordante foi encontrado em investigação, realizada em Rio Grande, estado do Rio Grande do Sul, visto que foi possível evidenciar que 193 (43,4\%) participantes tinham de 9 a 11 anos de estudo ${ }^{(12)}$. Já em Porto Velho, pesquisa acerca das barreiras à prevenção do CCU, desenvolvida com 286 mulheres, identificou que $64,8 \%$ delas possuíam entre 9 e 12 anos de estudo(15).

A deficiência no conhecimento sobre o exame citopatológico do colo do útero pode estar relacionada à baixa escolaridade das mulheres, o que pode contribuir para a não adesão a esse exame. No entanto, entre os resultados apresentados, não houve a realização de testes de associação entre essas variáveis. Mas pesquisa epidemiológica realizada em Feira de Santana, no estado na Bahia, constatou associação estatisticamente significativa entre não adesão ao exame e baixa escolaridade (valor de $p=0,01)^{(16)}$.

Em relação à renda mensal individual, grande parte das entrevistadas relatou não ter rendimento. Achado próximo foi identificado no município de Rio Grande - RS, em que boa parte das puérperas (340, 76,4\%) tinha renda familiar per capita menor que um salário mínimo. Tal investigação revelou ainda que essas mulheres e as que tiveram parto financiado pelo SUS apresentaram maior risco significativo de terem menor cobertura do exame citopatológico no final do pré-natal ${ }^{(12)}$. 
Diferentemente, em Bauru - SP, estudo desenvolvido com 370 mulheres assistidas em uma UBS, que objetivou avaliar o nível de informação sobre o exame citopatológico do colo do útero e sua associação com variáveis sociodemográficas, entre elas a renda, evidenciou que 192 (51,9\%) delas tinham renda familiar de até dois salários mínimos, e aquelas com maior renda apresentaram conhecimento adequado sobre o exame ${ }^{(17)}$.

A maioria das entrevistadas afirmou que o início da atividade sexual foi durante a adolescência, entre 15 e 19 anos. Achado semelhante foi identificado em Fortaleza, estado do Ceará, por meio de uma pesquisa em que predominou a faixa etária de 15 a 20 anos de idade, com $47(61,04 \%)$ relatos sobre o ano em que aconteceu a primeira relação sexual(18).

Outro estudo, também desenvolvido em Fortaleza, com 144 mulheres, sobre os fatores associados à realização de citologia para a prevenção do CCU, evidenciou que o início da prática sexual correspondeu a uma média de 17 anos de idade ${ }^{(19)}$. Essa variável deve ser investigada, porque o início precoce da atividade sexual constitui um fator de risco para o CCU, já que durante a adolescência as metaplasias se intensificam, e o coito aumenta a probabilidade de transformação atípica, potencializando as alterações celulares ${ }^{(20)}$.

A respeito de outro fator de risco para o CCU, foi investigado o histórico anterior de DST/IST, especialmente a infecção por HPV, e notou-se que esses agravos foram pouco frequentes. Entretanto, pode haver desconhecimento das entrevistadas sobre a sintomatologia das DST/IST. O mesmo achado foi observado em São Gonçalo do Amarante, estado do Ceará, em uma investigação em que foi aplicado o inquérito CAP, com 390 mulheres, pois apenas 39 (10\%) tiveram história pregressa de DST/IST ${ }^{(13)}$.

Observou-se que a maior parte das gestantes referiu ter de dois a três filhos. Diferentemente, no Rio de Janeiro, pesquisa realizada com 3.340 gestantes, sobre os riscos reprodutivos, mostrou que 1.151 $(35,5 \%)$ delas estavam na sua primeira gravidez ${ }^{(21)}$. Mas, concordando com o presente estudo, no Rio Grande - RS, verificou-se que 251 (56,4\%) mulheres referiram duas ou mais gestações, e 228 (51,2\%) eram multíparas; e o grupo de mulheres com duas a três gestações e partos prévios apresentou menor probabilidade de estar com o citopatológico desatualizado no fim do pré-natal ${ }^{(12)}$.

O conhecimento, a atitude e a prática das gestantes sobre o exame citopatológico do colo do útero revelaram-se majoritariamente inadequados. A falta de conhecimento sobre o exame e de sua finalidade, a vergonha, o medo, e o constrangimento, bem como a falta de atitude, o acesso limitado aos serviços de saúde e a assistência de saúde reduzida, são fatores que constituem barreiras para a realização do citopatológico, tornando as mulheres mais vulneráveis ao $\mathrm{CCU}^{(22)}$.

No que se refere à investigação do conhecimento das gestantes sobre o exame citopatológico do colo do útero, boa parte afirmou que já havia ouvido falar do exame $(\mathrm{N}=43)$ e que este pode ser realizado durante a gestação $(\mathrm{N}=31)$, mas não sabia a finalidade e não relatou pelo menos dois cuidados a serem tomados antes da sua realização. Logo, as participantes do presente estudo foram consideradas com conhecimento inadequado.

De modo contrário, no município de Turvânia, estado de Goiás, o conhecimento acerca do exame citopatológico do colo do útero, observado em 40 mulheres, foi elevado, pois todas o conheciam, e $32(80 \%)$ delas relataram a função de rastreamento do CCU do exame ${ }^{(23)}$. Em investigação com 453 mulheres de Floriano, estado do Piauí, sobre conhecimento, atitude e prática a respeito do exame citado, $181(36,7 \%)$ delas demonstraram ter conhecimento adequado ${ }^{(24)}$.

Por outro lado, e concordando com a presente pesquisa, em estudo fenomenológico heideggeriano com 10 gestantes, na ESF da Bahia, foi evidenciado que as mulheres atendem à solicitação do exame citopatológico do colo do útero, mas não compreendem o porquê da sua realização, visto que não souberam referir nenhuma relação do exame com a prevenção do $\mathrm{CCU}^{(25)}$.

A solicitação do exame citopatológico do colo do útero durante o período gestacional pode trazer muitas inquietações para as mulheres, uma vez que acreditam que ele não pode ser feito durante a gestação ${ }^{(5)}$. Pode-se ainda cogitar sobre a possibilidade de algumas mulheres preferirem não saber como é o exame, já que, no momento da sua realização, o profissional de saúde precisa observar uma região íntima, a qual, muitas vezes, elas próprias desconhecem e têm vergonha de olhar e tocar ${ }^{(26)}$. 
A respeito dos cuidados que devem ser realizados antes do exame, a maioria relatou que não sabia ou não se lembrava. Desconhecer esses cuidados significa não praticá-los, o que pode levar a alterações nos resultados e à dificuldade do procedimento em si e da leitura das lâminas, gerando problemas, como a frustração e a falta de retorno da mulher para exame ${ }^{(9)}$.

Em face disso, é importante que o profissional de saúde, em especial o enfermeiro, oriente as mulheres sobre os cuidados ou recomendações do Ministério da Saúde a serem tomados antes da realização do exame. São eles: evitar a utilização de lubrificantes, espermicidas ou medicamentos intravaginais por 48 horas, pois é utilizado gel no exame para a introdução do transdutor; abstinência sexual nos casos em que há o uso de lubrificantes e espermicidas e não estar menstruada, o que não se aplica às gestantes ${ }^{(2)}$.

Considerando-se que é importante a orientação para que as mulheres tenham conhecimento sobre o citopatológico e, consequentemente, atitude e prática adequadas, perguntou-se às participantes sobre as fontes de informação acerca do exame. Grande parte mencionou os familiares, e, entre esses, a maioria apresentava prática inadequada, o que leva à suposição de que eles também desconhecem o exame citopatológico do colo do útero e sua finalidade.

Diferentemente, estudo realizado com 267 mulheres, em São José de Mipibu, estado do Rio Grande do Norte, apresentou o médico como a principal fonte de informação sobre o exame citopatológico (105, $40,1 \%)$, seguido de amigas ou parentes $(53,20,2 \%)^{(27)}$. Os profissionais de saúde têm as ferramentas necessárias, por meio da educação em saúde, para dar empoderamento às usuárias sobre a prática do exame citopatológico ${ }^{(28)}$.

Em relação à atitude, as gestantes foram interrogadas ainda sobre a realização do exame durante a gestação, e 40 entrevistadas afirmaram que o realizariam, mas, quando indagadas sobre por que o fariam, relataram outros motivos que não a prevenção do CCU: detectar inflamação, prevenir doenças em geral, evitar infecção no feto ou durante o parto, proteger o bebê, saber se tem alguma alteração, e porque é importante. Resultado semelhante foi notado em pesquisa realizada com 10 gestantes, pois estas falaram sobre riscos e complicações que poderiam ter durante o parto ou outra patologia, mesmo aquelas que haviam realizado o exame durante a gestação ${ }^{(25)}$.

No que concerne à prática do exame citopatológico do colo do útero, a maioria não tinha realizado o exame durante a gravidez atual ou anterior, portanto, a prática foi considerada inadequada. Estudo qualitativo realizado com 13 gestantes, em Petrolina - $\mathrm{PE}$, com relação à atitude e também à prática sobre o exame citopatológico do colo do útero, mostrou que a indicação deste não significa prevenção. Ficou presente nos discursos que o exame não é realizado porque o profissional médico não o solicita e porque não deve ser realizado durante o período gestacional(5).

Assim, existe ainda a informação errônea de que o exame não deve ser realizado durante a gestação, o que demostra deficiência no conhecimento sobre o real motivo da sua realização e certa autoridade dos profissionais de saúde, pois ainda se entende que somente mediante a sua solicitação o exame pode ser realizado.

A aplicação do CAP permitiu um diagnóstico situacional, por identificação do conhecimento, atitude e prática das gestantes sobre o exame citopatológico do colo do útero, contribuindo para demonstrar fragilidades na população referida quanto ao rastreamento do CCU, o que evidencia a importância da construção de estratégias eficazes pelos enfermeiros.

\section{CONCLUSÃO}

Os resultados do estudo mostraram que a maior parte das gestantes apresentou conhecimento, atitude e prática inadequados sobre o exame citopatológico do colo do útero. Nesse contexto, é de grande valia definir estratégias supram as deficiências encontradas, de modo que essas mulheres possam ter mais conhecimento e adesão ao exame citopatológico do colo do útero, impactando positivamente a sua qualidade de vida com a detecção precoce do CCU e a redução da morbimortalidade por este agravo. 
Assim, sugere-se a formulação e a implantação de estratégias educativas pelos profissionais de saúde, em especial pelo enfermeiro, a fim de tornar as gestantes conhecedoras do exame citopatológico do colo do útero e permitir que elas sejam empoderadas quanto à atitude e prática adequadas para prevenção e detecção precoce do CCU.

É fundamental ainda ressaltar que durante a construção da presente pesquisa foi percebida uma séria limitação: a dificuldade em alcançar um quantitativo maior de gestantes, pois durante a coleta de dados notou-se pouco comparecimento das gestantes às UBS para o acompanhamento pré-natal.

\section{REFERÊNCIAS}

1. Instituto Nacional de Câncer José Alencar Gomes da Silva (BR). Coordenação de Prevenção e Vigilância Estimativa 2016. Síntese e resultado e comentários. [Internet] Rio de Janeiro: INCA; 2016 [acesso em 25 set 2016]. Disponível: www.inca.gov.br/wcm/dncc/2015/estimativa-2016.asp.

2. Ministério da Saúde (BR). Secretaria de Atenção à saúde. Departamento de Atenção básica. Atenção ao pré-natal de baixo risco. [Internet] Brasília: Ministério da Saúde; 2013 [acesso em 12 mar 2015]. Disponível: http://dab.saude.gov. br/portaldab/biblioteca.php?conteudo=publicacoes/cab32.

3. Instituto Nacional de Câncer José Alencar Gomes da Silva (BR). Coordenação de Prevenção e Vigilância. Estimativa 2014. Síntese de resultados e comentários. [Internet] Rio de janeiro: INCA; 2016 [acesso em 16 jun 2015]. Disponível: www.inca.gov.br/.../11-resenha-estimativa-2014-incidencia-de-cancer-no-brasil.pdf.

4. Oliveira MV, Guimarães MDC, França EB. Fatores associados a não realização de Papanicolaou em mulheres quilombolas. Ciênc. saúde coletiva. [Internet] 2014;19(11) [acesso em 15 mar 2016]. Disponível: http://dx.doi. org/10.1590/1413-812320141911.15642013.

5. Bezerra MWS, de Melo MCP, Moura LA, Moura JG, Cruz NM, Coelho RNM. Percepção de gestantes sobre o exame Papanicolaou: bases para a estratégia saúde da família. Rev. Ciên. Méd. Biol. [Internet] 2013;12(2) [acesso em 15 fev 2016]. Disponível: https://portalseer.ufba.br/index.php/cmbio/article/view/6962/6644.

6. Ministério da Educação (BR). Instituto Nacional do Desenvolvimento da Educação. Manual do aplicador do estudo CAP. [Internet] Brasília: Ministério da Educação; 2002 [acesso em 15 jul 2015]. Disponível: http://www.inde.gov.mz/ docs/monieduca10.doc.

7. Brenna SMF, Hardy E, Zeferino LC, Namura I. Conhecimento, atitude e prática do exame Papanicolaou em mulheres com câncer de colo uterino. Cad. Saúde Pública. [Internet] 2001;17(4) [acesso em 14 fev 2016]. Disponível: http:// dx.doi.org/10.1590/S0102-311X2001000400024.

8. Vasconcelos CTM. Efeitos de uma intervenção educativa na adesão das mulheres à consulta de retorno para receber o resultado do exame Papanicolaou [dissertação]. Fortaleza (CE): Universidade Federal do Ceará, 2008.

9. Neri EAR, Moura MSS, Penha JC, Reis TGO, Aquino PS, Pinheiro AKB. Conhecimento, atitude e prática sobre o exame Papanicolaou de prostitutas. Texto Contexto Enferm. [Internet] 2013;22(3) [acesso 20 fev 2016]. Disponível: http://dx.doi.org/10.1590/S0104-07072013000300020.

10. Conselho Nacional de Saúde. Resolução n.466, de 12 de dezembro de 2012. Dispõe sobre diretrizes e normas regulamentadoras de pesquisas envolvendo seres humanos. Brasília: Ministério da Saúde; 2013.

11. Conselho Nacional de Saúde. Resolução n.510, de 7 de abril de 2016. Dispõe sobre diretrizes e normas aplicáveis a pesquisas em ciências humanas e sociais. Brasília: Conselho de Saúde; 2016.

12. Gonçalves CV; Durate G, Costa JSD, Quintana SM, Marcolin AC. Perdas de oportunidades na prevenção do câncer de colo uterino durante o pré-natal. Ciênc. saúde coletiva. [Internet] 2011;16(5) [acesso em 20 fev 2016]. Disponível: http://dx.doi.org/10.1590/S1413-81232011000500020. 
13. Eduardo KGT, Moura ERF, Nogueira PSF, Costa CBJS, Pinheiro AKB, Silva RM. Conhecimento e mudanças e comportamento de mulheres junto a fatores de risco para o câncer de colo uterino. Rev. Rene. [internet] 2012;13(5) [acesso 20 fev 2016]. Disponível: http://www.periodicos.ufc.br/index.php/rene/article/view/4095.

14. Oliveira PSD, Lopes DA, Pinho L, da Silva Junior RFF, Oliveira HED, Barbosa HÁ. Membership of women to take preventive care from cervical cancer: a community trial. J Nurs UFPE on line. [Internet] 2016;10(2) [acesso em 25 abr 2017]. Disponível: http://www.revista.ufpe.br/revistaenfermagem/index.php/revista/article/view/8517/pdf_9532.

15. Rodrigues DE, Moreira KFA, de Oliveira TS. Barreiras à prevenção do câncer de colo uterino no município de porto Velho, Rondônia, Brasil. Invest. educ. enferm. [Internet] 2016;34(1) [acesso em 8 abr 2017]. Disponível: http://dx.doi. org/10.17533/udea.iee.v34n1a07.

16. Andrade, MS, de Almeida MMG, Araújo TM, Santos KOB. Fatores associados a não adesão ao Papanicolaou entre mulheres atendidas pela Estratégia Saúde da Família em Feira de Santana, Bahia, 2010. Epidemiol. Serv. Saúde. [Internet] 2014;23(1) [acesso em 16 nov 2017]. Disponível: http://dx.doi.org/10.5123/S1679-49742014000100011.

17. Leite MF, de Vitta FCF, Carnaz L, de Conti MHS, Marta SN, Gatti MAN et al. Conhecimento e prática das mulheres sobre o câncer de colo do útero de uma unidade básica de saúde. Journal of human growth and development. [Internet] 2014;24(2) [acesso em 9 2016]. Disponível: http://pepsic.bvsalud.org/scielo.php?pid=S010412822014000200014\&script=sci_ arttext\&tlng=p.

18. Diogenes MAR, Cesarino MCF, Jorge RJB, Queiroz INB, Mendes RS. Fatores de risco para o câncer cervical e adesão ao exame Papanicolaou entre trabalhadoras de enfermagem. Rev. Rene. [Internet] 2012;13(1) [acesso em 26 jun 2016]. Disponível: http://www.repositorio.ufc.br/handle/riufc/12874.

19. Falcão GB, Ibiapina FLP, Feitosa HN, Feitosa TS, de Lacerda PD, Braga JV, Carvalho FH. Fatores associados à realização de citologia para prevenção de câncer do colo uterino em uma comunidade urbana de baixa renda. Cad. saúde colet. [Internet] 2014;22(2) [acesso em 23 mar 2017]. Disponível: http://dx.doi.org/10.1590/1414-462X201400020009.

20. Lima CA, Palmeira JAV, Cipolotti R. Fatores associados ao câncer do colo uterino em Propriá, Sergipe, Brasil. Cad. Saúde Pública. [Internet] 2006;22(10) [acesso em 27 jul 2016]. Disponível: http://dx.doi.org/10.1590/S0102$311 \times 2006001000021$.

21. Xavier RB, Jannotti CB, da Silva KS, Martins AC. Risco reprodutivo e renda familiar: análise do perfil de gestantes. Ciên. saúde coletiva. [Internet] 2013;18(4) [acesso 29 dez 2016]. Disponível: http://dx.doi.org/10.1590/S141381232013000400029 .

22. Aguilar RP, Soares DA. Barreiras a realização do exame Papanicolaou: perspectivas de usuárias e profissionais da estratégia de saúde da família da cidade de Vitoria da Conquista-BA. Physis. [Internet] 2015;25(2) [acesso em 29 jul 2016]. Disponível: http://dx.doi.org/10.1590/S0103-73312015000200003.

23. Silva ECA, Dias MP, Fernandes CK, Nogueira DS, Barros EJ, Mota RM et al. Conhecimento das mulheres de 18 a 50 anos de idade sobre a importância do exame do câncer de colo uterino no município de Turvânia-GO. Rev. FMB. [Internet] 2015;8(4) [acesso em 30 nov 2016]. Disponível: http://revista.fmb.edu.br/index.php/fmb/article/view/181.

24. de Alburquerque CLF, Costa MP, Nunes FM, de Freitas RWJ, de Azevedo PRM, Fernandes JV et al. Knowledge, atitudes and pratices Reading the pap test among women in northeastern Brasil. São Paulo Med. J. [Internet] 2014;132(1) [acesso em 28 jul 2016]. Disponível: http://dx.doi.org/10.1590/1516-3180.2014.1321551.

25. Moreira RCR, Lopes RLM. Prevenção do câncer de colo do útero: desvelando a impessoalidade na voz das gestantes. Ciênc. cuid. saúde. [Internet] 2013;12(4) [acesso em 25 jul 2016]. Disponível: http://www.periodicos.uem.br/ojs/index. php/CiencCuidSaude/article/view/18973.

26. Peretto M, Drehmer LBR, Bello HMR. O não comparecimento ao exame preventivo do câncer de colo uterino: razões declaradas e sentimentos envolvidos. Cogitare Enferm. [Internet] 2012;17(1) [acesso em 25 abr 2017]. Disponível: http://dx.doi.org/10.5380/ce.v17i1.26371. 
27. Fernandes JV, Rodrigues SHL, Costa YGAS, Silva LC, Brito AML, Azevedo JWV et al. Conhecimento, atitude e pratica do exame Papanicolaou por mulheres do nordeste do Brasil. Rev. Saúde Pública. [Internet] 2009;43(5) [acesso 25 out 2016]. Disponível: http://dx.doi.org/10.1590/S0034-89102009005000055.

28. Ressel LB, Stumm KE, Rodrigues AP, dos Santos CC, Junges CF. Exame preventivo do câncer de colo uterino: a percepção das mulheres. AV. enferm. [Internet] 2013;31(2) [acesso em 26 ago 2016]. Disponível: http://revistas.unal. edu.co/index.php/avenferm/article/view/42766. 